\title{
Multi-valued solutions of a functional equation
}

\author{
by A. SMajdor and W. Smajdor (Katowice)
}

Abstract. The existence of a solution $\Phi_{0}$ of the inequality

$$
\Phi(x) \supset H(x, \Phi[f(x)])
$$

implies the existence of a minimal solution $\Phi \subset \Phi_{0}$ of the equation

(1)

$$
\Phi(x)=H(x, \Phi[f(x)])
$$

in some classes of multi-valued mappings. Moreover, if $f$ and $H$ are single-valued, then (under suitable conditions) the minimal continuum-valued solution of (1) is almost everywhere a singlevalued mapping.

Let $X$ be a non-empty set and let $Y$ be a topological Hausdorff space. We introduce the following classes of subsets of $Y$ :

$$
\begin{aligned}
& n(Y)=\{A \subset Y: A \neq \emptyset\}, \\
& c(Y)=\{A \subset Y: A \neq \emptyset \text { and } A \text { is compacit }\}, \\
& C(Y)=\{A \subset Y: A \neq \emptyset \text { and } A \text { is a continuum }\} .
\end{aligned}
$$

Let $Z$ be a topological space. We say that $\Phi: Z \rightarrow n(Y)$ is a closed mapping if, whenever $z_{0} \in Z, y_{0} \in Y, y_{0} \notin \Phi\left(z_{0}\right)$, there exist two open neighbourhoods $U\left(z_{0}\right)$ and $V\left(y_{0}\right)$ such that $\Phi(z) \cap V\left(y_{0}\right)=\emptyset$ for every $z \in U\left(z_{0}\right)$.

Let $z_{0} \in Z$. We say that $\Phi$ is upper semi-continuous (u.s.c.) at $z_{0}$ if for each open set $G$ containing $\Phi\left(z_{0}\right)$ there is an open neighbourhood $U\left(z_{0}\right)$ such that $\Phi(z) \subset G$ for every $z \in U\left(z_{0}\right)$.

A mapping $\Phi: Z \rightarrow n(Y)$ is u.s.c. if it is u.s.c. at every $z_{0} \in Z$.

We consider the following equation:

$$
\Phi(x)=H(x, \Phi[f(x)]),
$$

where the functions $f: X \rightarrow n(X), H: X \times Y \rightarrow n(Y)$ are given and $H(x, \cdot)$ is closed for every $x \in X$, and $\Phi: X \rightarrow n(Y)$ is the unknown function.

LEMMA 1. Let $F$ be a family of functions $\Phi: X \rightarrow c(Y)$ such that:

(I) if $\Phi \in F$, then $H(\cdot, \Phi[f(\cdot)]) \in F$ and $\Phi[f(x)] \in c(Y)$ for $x \in X$;

(II) if $\Phi_{n} \in F$ and $\Phi_{n+1} \subset \Phi_{n}$ for $n=1,2, \ldots$, then $\bigcap_{n=1}^{\infty} \Phi_{n} \in F$. 
If a function

(2)

$$
\Phi_{0} \in F
$$

fulfils the inequality

$$
\Phi_{0}(x) \supset H\left(x, \Phi_{0}[f(x)]\right)
$$

then the formulas

$$
\Phi_{n+1}=H\left(\cdot, \Phi_{n}[f(\cdot)]\right)
$$

and

$$
\bar{\Phi}=\bigcap_{n=1}^{\infty} \Phi_{n}
$$

define a solution $\bar{\Phi} \subset \Phi_{0}$ of (1) in the family $F$. If $\Psi: X \rightarrow n(Y)$ is a solution of (1) such that $\Psi \subset \Phi_{0}$, then $\Psi \subset \bar{\Phi}$.

Proof. It follows by (2), (I) and (4) that $\Phi_{n} \in F$ for $n=0,1,2, \ldots$ According to (2), (3) and (4) we have $\Phi_{n+1}(x) \subset \Phi_{n}(x)$ for $n=0,1,2, \ldots$ and for every $x \in X$. Therefore by (II) and (5) we get $\bar{\Phi} \in F$.

It is obvious that $H(x, \bar{\Phi}[f(x)]) \subset H\left(x, \Phi_{n}[f(x)]\right) \subset \Phi_{n}(x)$ for $n=0,1,2, \ldots$ and for $x \in X$. This implies the inclusion

$$
H(x, \bar{\Phi}[f(x)]) \subset \bar{\Phi}(x)
$$

for every $x \in X$.

Now, we take $z \in \bar{\Phi}(x)$. Then $z \in \Phi_{n+1}(x)=H\left(x, \Phi_{n}[f(x)]\right)$ for $n=0,1,2, \ldots$ There exists a sequence $\left\{y_{n}\right\}$ such that

$$
\mathrm{y}_{n} \in \Phi_{n}[f(x)] \subset \Phi_{0}[f(x)], \quad n=0,1,2, \ldots
$$

and

$$
z \in H\left(x, y_{n}\right)
$$

If $m \geqslant n$, then $y_{m} \in \Phi_{n}[f(x)]$. Thus

$$
A_{n}:=\mathrm{cl}\left\{y_{n}, y_{n+1}, \ldots\right\} \subset \Phi_{n}[f(x)], \quad n=0,1,2, \ldots,
$$

for the sets $\Phi_{n}[f(x)]$ are closed (being compact). We have $A_{n+1} \subset A_{n}, n$ $=0,1,2, \ldots$, and so there exists

$$
y \in \bigcap_{n=0}^{x} A_{n} .
$$

We shall prove that

$$
z \in H(x, y)
$$


Suppose, conversely, that $z \notin H(x, y)$. Then there exist two neighbourhoods $U$ and $V$ of points $y$ and $z$, respectively, such that

$$
H(x, U) \cap V=\varnothing \text {. }
$$

It follows by (8) that there exists $k, k=0,1,2, \ldots$, such that $y_{k} \in U$. Since by (6)

$$
z \in H\left(x, y_{k}\right) \cap V \subset H(x, U) \cap V,
$$

we have a contradiction. To prove the first part of the lemma it is sufficient to notice that according to (9), (8), (7) and (5) we have

$$
z \in H(x, y) \subset H\left(x, \bigcap_{n=0}^{x} A_{n}\right) \subset H\left(x, \bigcap_{n=0}^{x} \Phi_{n}[f(x)]\right)=H(x, \bar{\Phi}[f(x)]) .
$$

The second part of the lemma is obvious.

The set of all functions $\Phi: X \rightarrow n(Y)$ is partially ordered by the relation:

$$
\Phi_{1} \subset \Phi_{2} \quad \text { iff } \quad \Phi_{1}(x) \subset \Phi_{2}(x) \text { for every } x \in X .
$$

LEMMA 2. Let $F$ be a family of mappings $\Phi: X \rightarrow c(Y)$ such that conditions (I) of Lemma 1 and

(II') if $\Phi_{1} \in F$ for every $1 \in I$ and the family $\left\{\Phi_{i}\right\}_{1 \in I}$ is a chain, then $\bigcap_{1 \in I} \Phi_{1} \in F$ are fulfilled. Let a mapping $\Phi_{0} \in F$ fulfil inequality (3). Then there exists a minimal solution $\Phi \subset \Phi_{0}$ of equation (1) in the family $F$.

Proof. Let

$$
F_{0}:=\left\{\Phi \in F: \Phi \subset \Phi_{0}, \Phi \text { is a solution of (1) }\right\} .
$$

It follows by Lemma 1 that the family $F_{0}$ is not empty. Let $T$ be a chain in $F_{0}$, and let $\Phi_{T}=\bigcap_{\Phi \in T} \Phi$. According to $\left(\mathrm{II}^{\prime}\right), \Phi_{T} \in F$. Moreover, if $\Phi \in T$, then we have

$$
\Phi(x)=H(x, \Phi[f(x)]) \supset H\left(x, \Phi_{\tau}[f(x)]\right)
$$

and

$$
\Phi_{T}(x) \supset H\left(x, \Phi_{T}[f(x)]\right) .
$$

Lemma 1 guarantees the existence of a solution $\bar{\Phi}_{T} \subset \Phi_{T}$ of (1) in the family $F$. This shows that $\bar{\Phi}_{T}$ is a minorant of $T$ in $F_{0}$. The family $F_{0}$ possesses a minimal element in virtue of Kuratowski-Zorn lemma.

LeMmA 3. Let $X$ be a topological space and let $\Phi: X \rightarrow n(Y)$ be u.s.c. at $x_{0} \in X$. Then the mapping $x \rightarrow(x, \Phi(x))$ is u.s.c. at $x_{0}$.

Proof. Let $G$ be an open set in $Y$ such that $\Phi\left(x_{0}\right) \subset G$ and let $V$ be an open set in $X$ such that $x_{0} \in V$. Then there exists an open neighbourhood 
$U\left(x_{0}\right)$ of $x_{0}$ such that if $x \in U\left(x_{0}\right)$, then $\Phi(x) \subset G$, because $\Phi$ is u.s.c. at $x_{0}$. For every $x \in U\left(x_{0}\right) \cap V$ we have $(x, \Phi(x)) \subset V \times G$.

Lemma 4. Let $X$ be a topological space. If $\Phi: X \rightarrow n(Y)$ is a closed mapping, then the image $\Phi(K)$ of a compact subset $K$ of $X$ is a closed set.

Proof. We shall prove that the set $Y \backslash \Phi(K)$ is open. Let $y \notin \Phi(K)$. Then there exist neighbourhoods $V^{x}(y)$ and $U(x)$ such that

$$
\Phi(U(x)) \cap V^{x}(y)=\varnothing
$$

for every $x \in K$. Since the set $K$ is compact, there exist elements $x_{1}, x_{2}, \ldots, x_{n}$ in $K$ such that $K \subset U\left(x_{1}\right) \cup \ldots \cup U\left(x_{n}\right)$. Putting $V(y)=V^{x_{1}}(y) \cap \ldots$ $\ldots \cap V^{x_{n}}(y)$ we have

$$
\Phi(K) \cap V(y)=\emptyset .
$$

Therefore $Y \backslash \Phi(K)$ is open.

Definition. We say that equation $(1)$ has property $(P)$ in the family $F$ iff the following statement is true:

If $\Phi_{0} \in F$ is a solution of (3), then formulas (4) and (5) define a solution $\bar{\Phi} \subset \Phi_{0}$ of (1) in $F$ and there exists a minimal solution $\underline{\Phi} \subset \Phi_{0}$ of (1) in $F$.

THEOREM 1.1. If $f: X \rightarrow X$, then equation (1) has property (P) in the family of all functions $\Phi: X \rightarrow c(Y)$.

2. If $f: X \rightarrow X$ and $H$ with connected values is u.s.c. with respect to the second variable, then equation (1) has property (P) in the family of all functions $\Phi: X \rightarrow C(Y)$.

3. If $X$ is a topological Hausdorff space, $f: X \rightarrow c(X)$ is u.s.c. and $H$ is u.s.c., then equation (1) has property (P) in the family of all u.s.c. functions $\Phi: X \rightarrow c(Y)$.

4. If $X$ is a topological Hausdorff space, $f: X \rightarrow C(Y)$ is u.s.c. and $H$ with connected values is u.s.c., then equation (1) has property $(\mathrm{P})$ in the family of all u.s.c. functions $\Phi: X \rightarrow C(Y)$.

Proof. In order to prove this theorem we must test that suitable families fulfil conditions (I) and (II') of Lemmas 1 and 2.

1. Let $F_{1}=\left\{\Phi: \Phi \subset \Phi_{0}, \Phi: X \rightarrow c(Y)\right\}$, where $\Phi_{0}: X \rightarrow c(Y)$ is a given solution of (3). For every $\Phi \in F_{1}$ and $x \in X$ the sets $\Phi[f(x)]$ are compact and $H(x, \Phi[f(x)])$ are closed, as the images of compact sets by closed mappings. The inclusions

$$
H(x, \Phi[f(x)]) \subset H\left(x, \Phi_{0}[f(x)]\right) \subset \Phi_{0}(x)
$$

imply that $H(x, \Phi[f(x)]) \in c(Y)$. Hence

$$
H(\cdot, \Phi[f(\cdot)]) \in F_{1} .
$$


We suppose that $\left\{\Phi_{1}\right\}_{t \in l}$ is a chain in $F_{1}$. Then $\Phi(x)=\bigcap_{i \in I} \Phi_{1}(x) \in \mathcal{c}(Y)$ and $\Phi \subset \Phi_{0}$. Therefore conditions (I) and $\left(\mathrm{II}^{\prime}\right)$ of Lemmas 1 and 2 are fulfilled. The first part of the theorem is proved.

2. Let $f: X \rightarrow X$ and let $H$ with connected values be u.s.c. with respect to the second variable. Let $F_{2}=\left\{\Phi: \Phi \subset \Phi_{0}, \Phi: X \rightarrow C(Y)\right\}$, where $\Phi_{0}: X \rightarrow C(Y)$ is a solution of (3). The image of a connected set by an u.s.c. mapping with connected values is connected (cf. [4]). The set $H(x, \Phi[f(x)])$ is closed by Lemma 4, and by the inclusions (10) it is compact. Thus we have $H(\cdot, \Phi[f(\cdot)]) \in F_{2}$. The family $F_{2}$ has property (II'), because an intersection of a chain of continuums is a continuum (cf. [3]).

3. We suppose that $X$ is a topological Hausdorff space, $f: X \rightarrow c(X)$ is u.s.c. and $H$ is u.s.c.. Let $F_{3}=\left\{\Phi: \Phi \subset \Phi_{0}, \Phi: X \rightarrow c(Y), \Phi\right.$ is u.s.c. $\}$, where $\Phi_{0}: X \rightarrow c(Y)$ is an u.s.c. solution of (3). The set $\Phi[f(x)]$ is compact for all $x$ because $\Phi$ is an u.s.c. mapping with compact values and $f(x)$ is compact. Since the composition of two u.s.c. mappings is an u.s.c. mapping (cf. [2]), then by Lemmas 3 and 4 and by (10) we get $H(\cdot, \Phi[f(\cdot)]) \in F_{3}$. The family $F_{3}$ has property (II') because the intersection of a chain of compact sets is compact and the intersection of a family of u.s.c. mappings with compact values is u.s.c. (cf. [2]).

4. We suppose that $X$ is a topological Hausdorff space, $f: X \rightarrow C(X)$ is u.s.c. and $H$ with connected values is u.s.c. Let $F_{4}=\left\{\Phi: \Phi \subset \Phi_{0}, \Phi\right.$ : $X \rightarrow C(Y), \Phi$ is u.s.c. $\}$, where $\Phi_{0}: X \rightarrow C(Y)$ is an u.s.c. solution of (3). The family $F_{4}$ is included in $F_{3}$. It follows by the connectedness of $H(x, \Phi[f(x)])$ that $H(\cdot, \Phi[f(\cdot)]) \in F_{4}$. The family $F_{4}$ has property (II'), because the intersection of a chain of continuums is a continuum.

THEOREM 2. Let $f: X \rightarrow X$ and $H: X \times \boldsymbol{R} \rightarrow \boldsymbol{R}$ be continuous and increasing with respect to the second variable. The function $\Phi: X \rightarrow C(R)$ is a solution of (1) if and only if $\varphi(x)=\min \Phi(x)$ and $\psi(x)=\max \Phi(x)$ fulfil (1).

Proof. Necessity. The following equalities hold according to continuity and monotonicity of $H$ with respect to the second variable and by (1):

$$
\begin{aligned}
{[H(x, \varphi[f(x)]), H(x, \psi[f(x)])] } & =H(x,[\varphi[f(x)], \psi[f(x)]]) \\
& =H(x, \Phi[f(x)])=\Phi(x)=[\varphi(x), \psi(x)] .
\end{aligned}
$$

Thus $H(x, \varphi[f(x)])=\varphi(x)$ and $H(x, \psi[f(x)])=\psi(x)$. Hence $\varphi$ and $\psi$ are solutions of (1).

The proof of the sufficiency is also easy.

As a corollary we get the following

THEOREM 3. Let $f: X \rightarrow X$ and $H: X \times \boldsymbol{R} \rightarrow \boldsymbol{R}$ be an increasing and continuous function with respect to the second variable. If $\Phi_{0}: X \rightarrow C(R)$ is a solution of (3), then there exist a minimal solution $\Phi: X \rightarrow C(\boldsymbol{R})$ of (1), $\Phi \subset \Phi_{0}$, and this solution is a single-valued mapping. 
We have by a direct verification

Lemma 5. Let $X$ be a topological space. If a mapping $\Phi: X \rightarrow c(\boldsymbol{R})$ is us.c., then $\varphi(x)=\min \Phi(x)$ is lower semi-continuous and $\psi(x)=\max \Phi(x)$ is upper semi-continuous. $A$ mapping $\Phi: X \rightarrow C(R)$ is u.s.c. if and only if $\varphi(x)=\min \Phi(x)$ is lower semi-continuous and $\psi(x)=\max \Phi(x)$ is upper semi-continuous.

Definition. Let $X$ and $Y$ be topological spaces and let $\Phi: X \rightarrow n(Y)$ be a mapping. The closure of $\Phi$ is the mapping $\operatorname{cl} \Phi$ defined by

$$
\operatorname{cl} \Phi(x)=\left\{y \in Y:(x, y) \in \operatorname{cl} \Gamma_{\Phi}\right\} \quad \text { for } x \in X,
$$

where $\Gamma_{\Phi}$ is the graph of $\Phi$.

If $X$ and $Y$ are metric spaces, the above definition in equivalent to the condition:

$y \in \operatorname{cl} \Phi(x)$ if and only if there exist sequences $\left\{x_{n}\right\}$ and $\left\{y_{n}\right\}$ such that $x_{n}$ $\rightarrow x, y_{n} \rightarrow y$ and $y_{n} \in \Phi\left(x_{n}\right)$ for every $n$.

Lemma 6. Let $X$ be a topological space. Then the mapping $\mathrm{cl} \Phi$ is closed for every mapping $\Phi: X \rightarrow n(Y)$.

Pro of. Let $y_{0} \notin \operatorname{cl} \Phi\left(x_{0}\right)$. Then $\left(x_{0}, y_{0}\right) \notin \operatorname{cl} \Gamma_{\phi}$. There exist neighbourhoods $U\left(x_{0}\right)$ and $V\left(y_{0}\right)$ such that

$$
U\left(x_{0}\right) \times V\left(y_{0}\right) \cap \Gamma_{\Phi}=\varnothing .
$$

We shall prove that

$$
\operatorname{cl} \Phi(x) \cap V\left(y_{0}\right)=\varnothing
$$

for every $x \in U\left(x_{0}\right)$. Let $z \in \operatorname{cl} \Phi(x) \cap V\left(y_{0}\right)$ for some $x \in U\left(x_{0}\right)$. Then $(x, z) \in \operatorname{cl} \Gamma_{\Phi}$. There exist points $x^{\prime} \in U\left(x_{0}\right)$ and $z^{\prime} \in V\left(y_{0}\right)$ such that $\left(x^{\prime}, z^{\prime}\right) \in \Gamma_{\Phi}$. Thus $\left(x^{\prime}, z^{\prime}\right) \in U\left(x_{0}\right) \times V\left(y_{0}\right) \cap \Gamma_{\phi}$, but this is imposible by (11).

Lemma 7. Let $X$ be a topological space and let $Y$ be compact. If $H: X \times Y$ $\rightarrow Y$ is continuous, $f$ is an open and continuous function from $X$ into $X$ and if $\Phi: X \rightarrow n(Y)$ is a solution of (1), then $\operatorname{cl} \Phi$ is an u.s.c. solution of (1) with compact values.

Proof. The set $\operatorname{cl} \Phi(x)$ is closed, for the mapping $\operatorname{cl} \Phi$ is closed. Thus $\operatorname{cl} \Phi$ has compact values and is u.s.c. The mapping $\operatorname{cl} \Phi \circ f$ is u.s.c. as the composition of u.s.c. mappings. Further, $H(\cdot, \mathrm{cl} \Phi[f(\cdot)])$ is u.s.c. by Lemma 3 and by the continuity of $H$. The inclusion

$$
\Phi(x) \subset H(x, \operatorname{cl} \Phi[f(x)])
$$

implies

$$
\operatorname{cl} \Phi(x) \subset \operatorname{cl} H(x, \operatorname{cl} \Phi[f(x)])=H(x, \operatorname{cl} \Phi[f(x)]),
$$

since an u.s.c. mapping with compact values is closed. Now, we shall prove. the inclusion 


$$
H(x, \operatorname{cl} \Phi[f(x)]) \subset \operatorname{cl} \Phi(x)
$$

Let

$$
y \in H(x, \operatorname{cl} \Phi[f(x)])
$$

and let $U$ and $V$ be any neighbourhoods of points $x$ and $y$, respectively. It follows by (13) that there exists

$$
z \in \operatorname{cI} \Phi[f(x)]
$$

such that

$$
y=H(x, z) .
$$

There exist neighbourhoods $U_{1} \subset U$ and $W$ of points $x$ and $z$, respectively, such that

$$
H\left(U_{1} \times W\right) \subset V .
$$

The function $f$ is open; thus the set $f\left(U_{1}\right)$ is a neighbourhood of $f(x)$. Condition (14) implies

$$
\Phi\left[f\left(U_{1}\right)\right] \cap W \neq \emptyset \text {. }
$$

Hence, there exist $u \in U_{1}$ and $w \in W$ such that $w \in \Phi[f(u)]$. Consequently

$$
H(u, w) \in H(u, \Phi[f(u)])=\Phi(u)
$$

and

$$
H(u, w) \in H\left(U_{1} \times W\right) \subset V .
$$

Thus

$$
\Phi(U) \cap V \neq \varnothing
$$

which completes the proof of (12).

LEMMia 8. Let $X$ be a topological space, $f: X \rightarrow X$ and let $H: X \times \boldsymbol{R} \rightarrow \boldsymbol{R}$ be continuous and increasing with respect to the second variable. If $\Phi$ : $X \rightarrow c(R)$ is a solution of $(1)$, then $\hat{\Phi}(x):=\operatorname{conv}[\Phi(x)]$ is a solution of (1) with compact and connected values. Moreover, if $\Phi$ is u.s.c., then $\hat{\Phi}$ is u.s.c., too.

Proof. We define $\varphi(x)=\min \Phi(x), \psi(x)=\max \Phi(x)$. By (1) and by the monotonicity of $H$ with respect to the second variable we have

$$
\begin{aligned}
\hat{\Phi}(x) & =\operatorname{conv}[\Phi(x)]=\operatorname{conv}[H(x, \Phi[f(x)])] \\
& =[H(x, \varphi[f(x)]), H(x, \psi[f(x)])] .
\end{aligned}
$$

Since $\hat{\Phi}(x)=[\varphi(x), \psi(x)]$, we have

$$
\begin{aligned}
H(x, \hat{\Phi}[f(x)]) & =H(x,[\varphi[f(x)], \psi[f(x)]]) \\
& =[H(x, \varphi[f(x)]), H(x, \psi[f(x)])],
\end{aligned}
$$


by the continuity and monotonicity of $H$ with respect to the second variable. From (15) and (16) we get

$$
\hat{\Phi}(x)=H(x, \hat{\Phi}[f(x)]) .
$$

Lemma 5 implies that, if $\Phi$ is an u.s.c. function, then $\varphi$ is lower semicontinuous and $\psi$ is an upper semi-continuous numerical function. Thus $\hat{\Phi}(x)=[\varphi(x), \psi(x)]$ is u.s.c. by Lemma 5 .

TheOREM 4. Let $X$ be a metric space and let $f: X \rightarrow X$ be a continuous and open function $X$ into $X$. Moreover, let a continuous function $H: X \times$ $\times \boldsymbol{R} \rightarrow \boldsymbol{R}$ be increasing with respect to the second variable. If $\Phi_{0}: X \rightarrow C(\boldsymbol{R})$ is an u.s.c. solution of inequality (3), and if there exists a compact set $K \subset \boldsymbol{R}$ such that $\Phi_{0}(X) \subset K$, then a minimal u.s.c. solution $\Phi: X \rightarrow C(R)$ of (1) such that $\Phi \subset \Phi_{0}$ is almost everywhere (everywhere except a set of the first category) a single-valued mapping.

Proof. The existence of a minimal u.s.c. solution $\Phi: X \rightarrow C(R)$ with $\Phi \subset \Phi_{0}$ is guaranteed by Theorem 1. If $\Phi(x)=[\varphi(x), \psi(x)]$, then (by Theorem 2) the functions $\varphi$ and $\psi$ fulfil equation (1). According to Lemma 7 the functions $\operatorname{cl} \varphi$ and $\operatorname{cl} \psi$ are u.s.c. solutions of (1). Lemma 8 implies that $\Phi_{1}(x)=\operatorname{conv}[\operatorname{cl} \varphi(x)]$ and $\Phi_{2}(x)=\operatorname{conv}[\operatorname{cl} \psi(x)]$ are u.s.c. solution of (1) and $\Phi_{1}: X \rightarrow C(R), \Phi_{2}: X \rightarrow C(R)$. By the minimality of $\Phi$ we have $\Phi_{1}=\Phi_{2}=\Phi$. The equality $\operatorname{conv}[\operatorname{cl} \varphi(x)]=[\varphi(x), \psi(x)]$ implies that $\psi(x) \in \operatorname{cl} \varphi(x)$. This means that there exist sequences $\left\{y_{n}\right\},\left\{x_{n}\right\}$ such that $y_{n}$ $\rightarrow \psi(x), x_{n} \rightarrow x$, and $y_{n}=\varphi\left(x_{n}\right)$. Thus there exists a sequence $\left\{x_{n}\right\}$ such that $x_{n} \rightarrow x$ and $\varphi\left(x_{n}\right) \rightarrow \psi(x)$. If $\varphi$ is continuous at $x$, then $\varphi(x)=\psi(x)$. In virtue of the Baire theorem the set of all $x$ at which $\varphi$ is not continuous is of the first category.

Let $\Phi_{0}$ be an u.s.c. solution of (3) with values in $C(\boldsymbol{R})$. K. Baron in [1] proved (under suitable assumptions) the existence of a single-valued u.s.c. solution $\Phi$ of (1) such that $\Phi \subset \Phi_{0}$. The following example indicates that the assumptions of Theorem 4 do not ensure the existence of an u.s.c. singlevalued solution $\Phi$ of (1) such that $\Phi \subset \Phi_{0}$.

Example. Let $X=Y=R, f(x)=2 x, H(x, y)=y$ and let $\Phi_{0}$ be given by the formula

$$
\Phi_{0}(x)= \begin{cases}0 & \text { for } x<0, \\ {[0,1]} & \text { for } x=0, \\ 1-\left|2^{n+2} x-3\right| & \text { for } x \in\left[2^{-(n+1)}, 2^{-n}\right], n=0, \pm 1, \pm 2, \ldots\end{cases}
$$

This mapping is an u.s.c. solution of (1) with compact and connected values. Thus $\Phi_{0}$ fulfils (3), but equation (1) does not possess any u.s.c. single-valued solution. 


\section{References}

[1] $\mathrm{K}$. Barom, On the continuous solutions of a non-linear functional equation of the first order, Ann. Polon. Math. 28 (1973), p. 201-205.

[2] C. Berge, Topological spaces, Edinburgh and London 1963.

[3] R. Engelking, General topology, Warszawa 1977.

[4] R. E. Smithson, Multifunction, Nieuw. Arch. Wisk. 20 (1972), p. 31-53.

Reçu par la Rédaction le 1.12.1977 